\title{
Numerical Study of Competing Spin-Glass and Ferromagnetic Order
}

\author{
M.V. Simkin \\ Department of Physics, Brown University, \\ Providence, RI 02912-1843
}

\begin{abstract}
Two and three dimensional random Ising models with a Gaussian distribution of couplings with variance $J$ and non-vanishing mean value $J_{0}$ are studied using the zero-temperature domain-wall renormalization group (DWRG). The DWRG trajectories in the $\left(J_{0}, J\right)$ plane after rescaling can be collapsed on two curves: one for $J_{0} / J>r_{c}$ and other for $J_{0} / J<r_{c}$. In the first case the DWRG flows are toward the ferromagnetic fixed point both in two and three dimensions while in the second case flows are towards a paramagnetic fixed point and spin-glass fixed point in two and three dimensions respectively. No evidence for an extra phase is found.
\end{abstract}

In some range of concentration of magnetic impurities in a non-magnetic host one observes a competition between spin-glass and ferromagnetic order [1]. The phenomenon can be described by an Ising model in which couplings are distributed randomly with some non-vanishing mean value $J_{0}$ and width $J$. This issue has been addressed by Sherrington and Kirkpatrick [2] in the case of a soluble infinite-range model. For the case of insoluble short range model Migdal-Kadanoff real-space rescaling [3], [4] and computer simulations [5]- [13] have been employed. It is established [8], [9] that there is no finite-temperature phase transition in a two-dimensional (2d) Ising spin-glass (random Ising model with $J_{0}=0$ ). McMillan 77 investigated the $2 \mathrm{~d}$ random Ising model for the general case, $J_{0}$ not necessarily zero, using finite-temperature domain-wall renormalization group (DWRG). He found at small temperatures only two phases: ferromagnetic and paramagnetic. However, some later studies [12], [13] have found a finite temperature transition to a "random antiphase state", which has similar properties to a spin glass. Therefore more study of the subject is desirable. To my knowledge no DWRG study of three dimensional (3d) random Ising model (with $J_{0} \neq 0$ ) have been done. In this paper the $2 \mathrm{~d}$ and $3 \mathrm{~d}$ random Ising models are investigated using the zero-temperature DWRG [7]-[9]. In particular no evidence for the "random antiphase state" is found.

The system is the Edwars-Anderson model [14] of an Ising spin glass with Hamiltonian:

$$
H=-\sum_{i j} J_{i j} S_{i} S_{j}
$$

where $S_{i}= \pm 1, J_{i j} \neq 0$ only when lattice sites $i, j$ are nearest neighbors. Each coupling constant $J_{i j}$ is an independent Gaussian distributed random variable with $P\left(J_{i j}\right)=\frac{1}{\sqrt{2 \pi J^{2}}} \exp \left(-\left(J_{i j}-J_{0}\right)^{2} / 2 J^{2}\right)$. The lattices studied are $d$-dimensional square $(d=2)$ and simple cubic $(d=3)$ lattices of linear size $L$. The zero-temperature DWRG method [8]-[9] was used by computing the ground-state energies for periodic and antiperiodic boundary conditions (BC) in one direction, with free $\mathrm{BC}$ in the other $d-1$ directions. The difference $\Delta E(L) \equiv E_{p}(L)-E_{a p}(L)$ is the domain-wall energy which may be interpreted as an effective coupling constant on scale $L . \Delta E(L)$ has sample to sample fluctuations and one can define the mean $J_{0}(L)$ and the width $J(L)$ at scale $L$ by

$$
J(L)=\sqrt{J_{0}(L)=<\Delta E(L)>},
$$


where $\langle\ldots\rangle$ is the average over samples with different realizations of the coupling constants $\left\{J_{i j}\right\}$. The ground state energy is found by simulated annealing [15]. Each annealing was start from random spin configuration or $T=\infty$. The temperature was reduced in $n_{s}$ steps from $T_{h}=5$ to $T_{l}=0.05$ by a factor $10^{-2 / n_{s}}$. At each intermediate temperature $T_{n}=T_{h} 10^{-2 n / n_{s}}$ the spins are updated by a single trial flip using the Metropolis algorithm. As a single annealing is not guaranteed to reach the minimum energy state, this procedure was repeated $n_{a}$ times, with the required CPU time proportional to $n_{a} \times n_{s}$. It is found empirically that the lowest energies for fixed CPU time are found when $n_{a} \simeq n_{s}$. This annealing schedule is substantially better than a large number of instantaneous quenches, for the same CPU time. To take maximum advantage of vectorization 64 annealings were performed simultaneously. To estimate the necessary values of $n_{a}$ and $n_{s}$ a few samples are tested until increasing $n_{a}$ and $n_{s}$ no longer leads to lower energy. Even so there is no guarantee that theses values of $n_{a}$ and $n_{s}$ are sufficient for all of the samples. To estimate the errors due to this, two runs for the same set of one hundred samples with periodic BC but with different sequences of random numbers for the Metropolis spin updating are performed. The two energy minima $E_{1}, E_{2}$ found in the two nominally identical annealings were recorded. If true absolute minima are found than $E_{1}=E_{2}$ but, if the true minima are not reached, then $<\left(E_{1}-E_{2}\right)^{2}>$ is a measure of the error due to not finding the exact minimum. The numbers $n_{a}$ and $n_{s}$ are then increased until this error is less than statistical error due to the finite number of samples, i.e. until $\delta E / E<N^{-1 / 2}$. All data is obtained for $N=10^{4}$ and the number of annealings $n_{a}$ and number of steps $n_{s}$ ranged from $n_{a}=64, n_{s}=10$ for the smallest sizes $(\mathrm{L}=2)$ to $n_{a}=192, n_{s}=200$ for the largest $(L=9$ in $d=2, L=5$ in $d=3)$.

The results of the simulations are shown in Fig. 1 as renormalization group flows in the $\left(J_{0}, J\right)$ plane. All initial couplings $J_{0}(1)$ and $J(1)$ are on the $J_{0}+J=1$ line from which the trajectories, indicated by dashed lines, start. Next symbols on the trajectories correspond to $L=2,3 \ldots$.

All the trajectories collapse into two curves by $L$ - independent rescaling

$$
\begin{aligned}
J_{0}(L) & \rightarrow \lambda(r) J_{0}(L) \\
J(L) & \rightarrow \lambda(r) J(L) .
\end{aligned}
$$

In Eq.3 the rescaling factor $\lambda(r)$ depends only on the ratio $r \equiv J_{0} / J$, and upon performing this rescaling all the flows of Fig.1 collapse on to the flows of Fig.2. That the rescaling of Eq. 3 works means that (at zero temperature) on any length scale $L$ the system is described by the Hamiltonian of Eq. 1 and no other terms are generated by the RG transformation.

The flows for $r>r_{c}$ seem to be flowing to a ferromagnetic fixed point with $J(L) / J_{0}(L)$ decreasing and $J_{0}(L)$ increasing with increasing $L$ and one may speculate that the $L=\infty$ fixed point is at $J(\infty) / J_{0}(\infty)=$ $0, J_{0}(\infty)=\infty$. For $r<r_{c}$, on the other hand, the flows would seem to be different in $d=2$ and $d=3$. In $d=2$ the flow is shown in Fig.2(a) and it seems that as $L$ increases both $J_{0}(L)$ and $J(L)$ ultimately decrease to zero with $J(L) / J_{0}(L) \rightarrow \infty$. This would be interpreted as a paramagnetic fixed point for any finite temperature and a spin-glass fixed point at $T=0$. In $d=3$ (see Fig.2(b)) the couplings $J_{0}(L) \rightarrow 0$ and $J(L) \rightarrow \infty$ which has the interpretation of a pure spin-glass fixed point. This spin-glass at $T=0$ is believed to survive at low temperatures $T<T_{c}$ in $d=3$ 10. Note that the critical value of $r=J_{0} / J$ is $r_{c}^{2 d} \cong 1.05$ and $r_{c}^{3 d} \cong 0.7$ which is in fairly good agreement with the values $r_{c}^{2 d} \cong 1.2$ and $r_{c}^{3 d} \cong 0.65$ obtained by a Migdal-Kadanoff RG method [4]. The value of $r_{c}^{2 d}$ is in agreement with the result of McMillan [7], who extrapolated his finite-temperature DWRG data to $T=0$ and got $r_{c}^{2 d} \cong 1.04$.

In a study of a related two-dimensional Ising model [12], 113] with a bimodal distribution $P\left(J_{i j}\right)=$ $p \delta\left(J_{i j}-1\right)+(1-p) \delta\left(J_{i j}+1\right)$ it was suggested that $J_{0}(L)$ and $J(L)$ scale as

$$
\begin{aligned}
& J_{0}(L) \sim L^{a} \\
& J(L) \sim L^{\tilde{a}},
\end{aligned}
$$

where for some range of $p, a<0$ and $\tilde{a}>0$ (see [12],Fig. 9). This was interpreted as evidence for a "random antiphase" state with properties similar to those of spin-glass. The raw data of Fig.1(a) seems to support the conjecture of Eq.4 as there are trajectories along which $J_{0}(L)$ and $J(L)$ seem to scale in 
this way. However, the simple additional rescaling of Eq.3 implies that the apparent $\tilde{a}>0$ is a finite size effect which disappears at large $L$.

In conclusion this $T=0$ DWRG study of random Ising models with $\left\langle J_{i j}\right\rangle=J_{0}>0$ indicates that, at low temperatures, there are only paramagnetic and ferromagnetic phases in $d=2$ and spin-glass and ferromagnetic in $d=3$. No sign of any other phase is seen. Unfortunately, simulated annealing becomes ineffective for large system sizes $L$, and computer limitations restricted $L$ to be quite small. However, recently it was found (Ref. [16]) that so-called branch and cut method allows one to study severall times larger systems than simulated annealing. Implementing this method for this particular problem may be a subject of future investigations.

I am grateful to J.M. Kosterlitz for useful conversations and to M.J.P. Gingras for correspondence. This work was supported by National Science Foundation Grant No. DMR-9222812. Computations were performed on Cray EL98 at the Theoretical Physics Computing Facility at Brown University.

\section{References}

[1] K.H. Fischer and J.A. Hertz, Spin Glasses (Cambridge University Press 1991); M.J.P. Gingras, in Magnetic Systems with Competing Interactions, ed. H.T. Diep, (World Scientific Publishing 1994).

[2] D. Sherrington and S. Kirkpatrick, Phys. Rev. Lett. 35, 1972 (1975).

[3] A.P. Young and R.B. Stinchcombe, J. Phys. C, 9, 4419 (1976).

[4] B.W. Southern and A.P. Young, J. Phys. C, 10, 2179 (1977).

[5] S. Kirkpatrick, Phys. Rev. B 16, 4630 (1977).

[6] I. Morgenstern and K. Binder, Phys. Rev. B 22, 288 (1980).

[7] W.L. McMillan, Phys. Rev. B 29, 4026 (1983).

[8] W.L. McMillan, Phys. Rev. B 30, 476 (1984).

[9] A.J. Bray and M.A. Moore, J. Phys. C, 17, L463 (1984).

[10] W.L. McMillan, Phys. Rev. B 31, 340 (1985); A.J. Bray and M.A. Moore, Phys. Rev. B 31, 631 (1985).

[11] T. Shirakura, O. Ono, S. Inawashiro, J. Phys. Soc. Jpn. 56, 3742 (1987).

[12] Y. Ozeki, J. Phys. Soc. Jpn. 59, 3531 (1990), and references therein.

[13] Y. Ueno and Y. Ozeki, J. Stat. Phys. 64, 227 (1991).

[14] S.F. Edwards and P.W. Anderson, J. Phys. F 5,965 (1975).

[15] S. Kirkpatrick, C. D. Gellat Jr, and M.P. Vecchi, Science, 220, 671 (1983).

[16] H. Rieger, L. Santen, U. Blasum, M. Diehl, M. Junger, and G. Rinaldi, J. Phys. A, 29, 3939 (1996); C. De Simone, M. Diehl, M. Junger, P. Mutzel, G. Reinelt, and G. Rinaldi, J. Stat. Phys. 80, 487 (1995). 

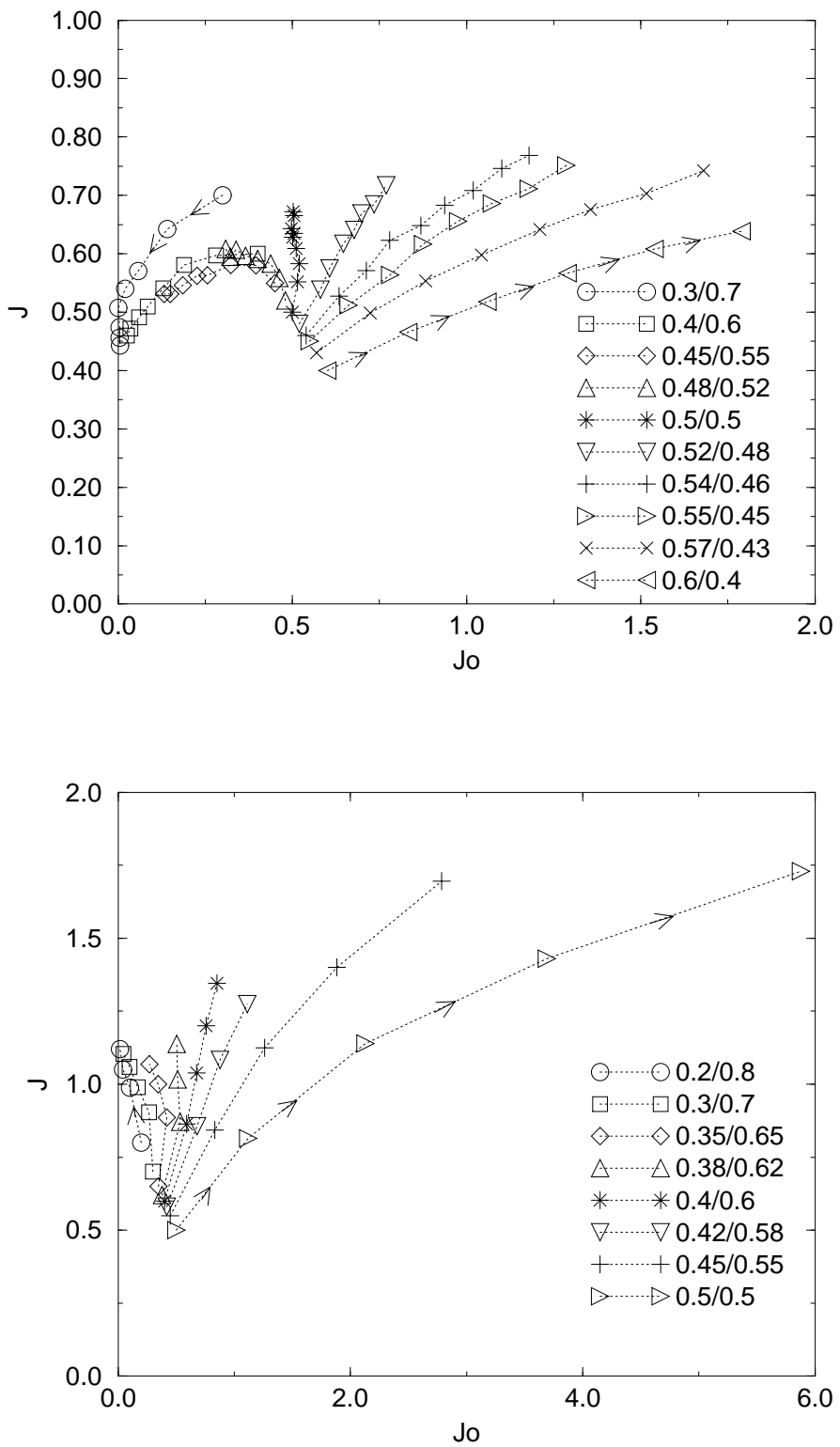

Figure 1: Domain wall renormalization group trajectories for two (a) and three (b) dimensional random Ising models in the mean value $\left(J_{0}\right)$ - variance $(J)$ plane. The trajectories, indicated by dashed lines, start on the line $J_{0}+J=1$. Direction of flows is indicated by arrows on some of the trajectoriies. The values of initial couplings $J_{0}$ and $J$ are indicated near corresponding symbols. Statistical uncertainty on each point is about the size of the symbol. 

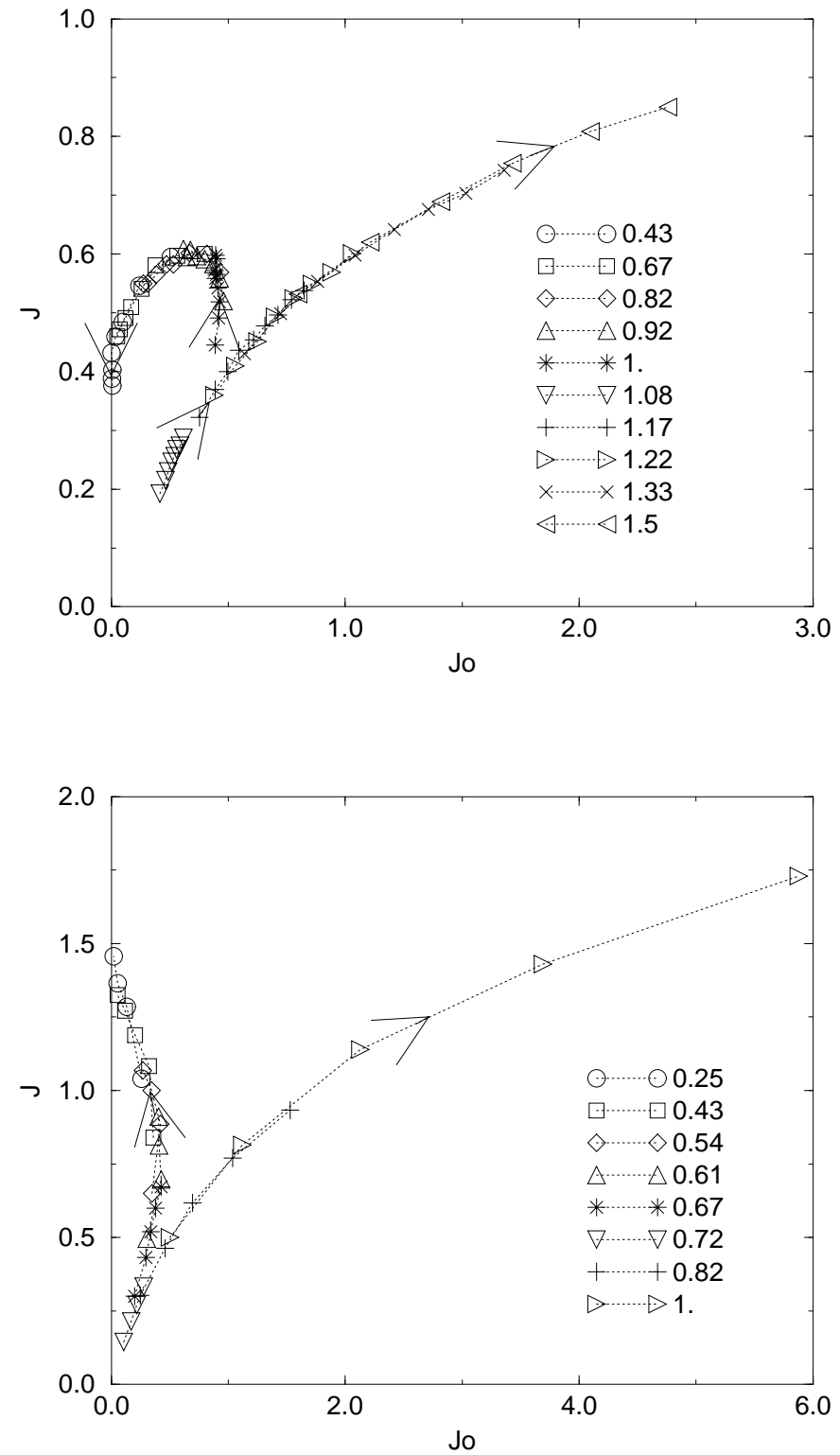

Figure 2: Rescaled, using Eq.3, renormalization group trajectories of $d=2$ (a) and $d=3$ (b) random Ising models. Instead of $J_{0}$ and $J$ as in Fig.1 their ratio is indicated near corresponding symbols. Trajectories for $J_{0} / J>r_{c}\left(r_{c}^{2 d}=1.05, r_{c}^{3 d}=0.7\right)$ flow to the ferromagnetic fixed point, while those for $J_{0} / J<r_{c}$ flow to paramagnetic fixed point in $2 \mathrm{~d}$ and spin-glass fixed point in $3 \mathrm{~d}$. 